\title{
Kalman conjecture for resonant second-order systems with time delay
}

DOI:

10.1109/cdc.2018.8619845

\section{Document Version}

Accepted author manuscript

Link to publication record in Manchester Research Explorer

\section{Citation for published version (APA):}

Zhang, J., Heath, W., \& Carrasco, J. (2019). Kalman conjecture for resonant second-order systems with time delay. In 57th IEEE Conference on Decision and Control 2018 https://doi.org/10.1109/cdc.2018.8619845

\section{Published in:}

57th IEEE Conference on Decision and Control 2018

\section{Citing this paper}

Please note that where the full-text provided on Manchester Research Explorer is the Author Accepted Manuscript or Proof version this may differ from the final Published version. If citing, it is advised that you check and use the publisher's definitive version.

\section{General rights}

Copyright and moral rights for the publications made accessible in the Research Explorer are retained by the authors and/or other copyright owners and it is a condition of accessing publications that users recognise and abide by the legal requirements associated with these rights.

\section{Takedown policy}

If you believe that this document breaches copyright please refer to the University of Manchester's Takedown Procedures [http://man.ac.uk/04Y6Bo] or contact uml.scholarlycommunications@manchester.ac.uk providing relevant details, so we can investigate your claim.

\section{OPEN ACCESS}




\title{
Kalman conjecture for resonant second-order systems with time delay
}

\author{
Jingfan Zhang, William P. Heath, Joaquin Carrasco
}

\begin{abstract}
We construct Zames-Falb multipliers for secondorder systems with time delay. There are at least two equality constraints on the multiplier phase in the limiting case as the damping ratio tends to zero and the gain approaches the Nyquist gain. Nevertheless we demonstrate a multiplier exists for every system we consider. Our results depend on numerical examples and searches; thus while the Kalman Conjecture is apparently verified for this class of system, a formal proof is beyond the scope of the paper.
\end{abstract}

\section{INTRODUCTION}

The Kalman conjecture [1] addresses the absolute stability of a Lur'e system [2] where the LTI plant is in negative feedback with a memoryless slope-restricted nonlinearity. While the Kalman conjecture is true for third-order plants [3], there are fourth-order counterexamples [4]. For a recent discussion, see [5].

In this paper we address Lur'e systems where the LTI system is second-order (with no zeros) but with delay, as in Figure 1. With time delay it remains an open question what class of system satisfies the Kalman conjecture [6]. On the one hand, a system with time delay, i.e. $G(s)=G_{n} e^{-s T_{d}}$ where $G_{n}$ is stable and rational, may be thought as a high order system, so it might seem reasonable that the Kalman conjecture may not be satisfied even when the order of $G_{n}(s)$ is low. On the other hand, it is straightforward to show, via the off-axis circle criterion, that first order systems with delay satisfy the Kalman conjecture. Moreover, we have introduced [7] a wide class of system where, if $G_{n}$ satisfies the Kalman conjecture, then $G(s)$ also satisfies the Kalman conjecture. In fact, to the authors' knowledge, there is no example in the literature where a general plant $G_{n}(s)$ satisfies the Kalman conjecture but $G(s)$ does not.

We can use multiplier theory to establish input-output stability; specifically, if a multiplier exists for a given LTI plant then the corresponding Lur'e system is absolutely stable. The Zames-Falb multipliers [8] are widest known class available for such analysis [9]. Recently several searches for Zames-Falb multipliers have been published [10]-[14]. Note that Zames-Falb multipliers are used both to establish that third-order plants satisfy the Kalman conjecture [3] and in the proof of the off-axis circle criterion [15] via phasesubstitution [17].

The class of system considered in [7] includes secondorder systems (with no zeros) with delay but without resonance (i.e. with damping ratio greater than $1 / \sqrt{2}$ ). Hence in

Control Systems Centre, School of Electrical and Electronic Engineering, University of Manchester, M13 9PL, UK.

jingfan. zhang@manchester.ac.uk

william.heath@manchester.ac.uk

joaquin.carrascogomez@manchester.ac.uk this paper we focus on systems where the damping ratio is less than $1 / \sqrt{2}$. Specifically we construct Zames-Falb multipliers for a wide variety of delay and damping ratio and hence show the Kalman conjecture to be true for each case. This requires construction of a Zames-Falb multiplier $M$ such that the phase of $M(j \omega)\left(1+k_{N} G(\omega)\right)$ is positive at all frequencies, where $k_{N}$ is the Kalman gain. Since by definition there is a frequency $\omega_{N}$ such that $1+k_{N} G\left(j \omega_{N}\right)=0$ it follows that the phases of $1+k_{N} G\left(j \omega_{N}^{+}\right)$and $1+k_{N} G\left(j \omega_{N}^{-}\right)$ differ by $180^{\circ}$. Hence the phase of $M\left(j \omega_{N}\right)$ is fixed; specifically we require

$$
\angle M\left(j \omega_{N}\right)+\left(\angle 1+k_{N} G\left(j \omega_{N}^{+}\right)+\angle 1+k_{N} G\left(j \omega_{N}^{-}\right)\right) / 2=0 .
$$

This puts a severe restriction on the available multipliers and renders the standard searches inapplicable. This is related to the restrictions found in [3] and the phase restriction for discrete-time multipliers discussed in [16]. For some delay values there are two frequencies where $1+k_{N} G(j \omega)=0$. In this case there are two frequencies where the phase of $M$ is fixed. We note that if these phases are different then the off-axis circle criterion cannot be applied.

The main part of this paper, Section III, focuses on the limiting case where the damping ratio tends to zero. This imposes a further frequency (specifically $\omega=1$ ) where the multiplier phase is fixed. In this sense the limiting case is the hardest for which to find multipliers. In Section IV we briefly discuss the case where the damping ratio is non-zero. In this case the limitations on the phase are less severe. However several quantities are no longer analytic necessitating further reliance on numerical results.

Although we do not offer a complete proof, we have not found any time-delayed second-order plant with no zeros where we cannot construct a suitable multiplier. We believe the Kalman conjecture to be true for such systems.

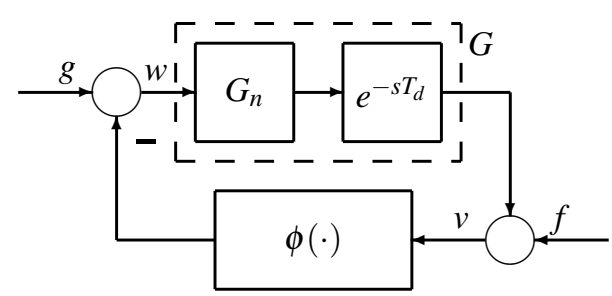

Fig. 1: Lur'e System with time delay. In this paper $G_{n}$ takes the structure $G_{n}(s)=\frac{1}{s^{2}+2 \xi s+1}$, while $\phi(\cdot)$ is a memoryless slope-restricted nonlinearity. 


\section{Preliminaries}

\section{A. Lur'e Systems}

Let $\mathbf{R H}_{\infty}$ be the space consisting of proper real rational transfer functions with no pole in the closed right-half complex plane. The notations $\mathfrak{R}\{G(j \omega)\}$ and $\mathfrak{I}\{G(j \omega)\}$ represent the real part and imaginary part of $G(j \omega)$ in the complex plane respectively. Throughout the text, units in time and frequency have been omitted.

Let $\mathbb{N}^{+}$be the set of non-negative integers, $\mathbb{R}^{+}$be the set of non-negative real numbers, and $\mathscr{L}_{2}\left(\mathbb{R}^{+}\right)$be the Hilbert space of all square integrable and Lebesgue measurable functions $f: \mathbb{R}^{+} \mapsto \mathbb{R}$. A truncation of the function $f$ at $T \in \mathbb{R}$ is given by $f_{T}(t)=f(t), \forall t \leq T ; f_{T}(t)=0, \forall t>T$. The function $f$ belongs to the extended space $\mathscr{L}_{2 e}\left(\mathbb{R}^{+}\right)$if $f_{T} \in \mathscr{L}_{2}\left(\mathbb{R}^{+}\right)$for all $T>0$.

A nonlinearity $\phi: \mathscr{L}_{2 e}\left(\mathbb{R}^{+}\right) \mapsto \mathscr{L}_{2 e}\left(\mathbb{R}^{+}\right)$is said to be memoryless if there exists a map $N: \mathbb{R} \rightarrow \mathbb{R}$ such that $(\phi(v))(t)=N(v(t)), \forall t \in \mathbb{R}$. The nonlinearity $\phi$ is said to be slope restricted, denoted by $\phi \in S[0, k]$, if $0 \leq N\left(x_{1}\right)-$ $N\left(x_{2}\right) \leq k\left(x_{1}-x_{2}\right), \forall x_{1}, x_{2} \in \mathbb{R}$ and $x_{1} \neq x_{2}$. The slope restricted nonlinearity is also sector bounded, but the reverse is not necessarily true.

The Lur'e system in Fig. 1 is given by

$$
v=f+G w, \quad w=g-\phi(v),
$$

where the signals are in the extended space $\mathscr{L}_{2 e}\left(\mathbb{R}^{+}\right)$. The feedback interconnection is said to be well-posed if the inverse map $(v, w) \mapsto(g, f)$ is causal in $\mathscr{L}_{2 e}\left(\mathbb{R}^{+}\right)$. Additionally, the interconnection is said to be stable if $(v, w)$ are in $\mathscr{L}_{2}\left(\mathbb{R}^{+}\right)$ for any $(g, f)$ in $\mathscr{L}_{2}\left(\mathbb{R}^{+}\right)$.

Definition 1 (Nyquist value, $k_{N}$ ): The Nyquist value of a stable transfer function $G(s)$ is

$$
k_{N}=\sup _{k}\left\{k>0:(1+\tau k G(s))^{-1} \text { is stable } \forall \tau \in[0,1]\right\} .
$$

Conjecture 1 (Kalman Conjecture [1]): Let $\phi$ be a memoryless nonlinearity, and $\phi \in S[0, k]$. The feedback interconnection between $G$ and $\phi$ is asymptotically stable if $k<k_{N}$.

\section{B. Zames-Falb Multipliers}

As our aim is to show that the delay does not change the behaviour of second order systems, we restrict our attention to the class of Zames-Falb multipliers that preserve the positivity for any $\phi \in S[0, k]$.

Definition 2 (Zames-Falb multiplier [8], [9]): The convolution operators $M: \mathscr{L}(\mathbb{R}) \mapsto \mathscr{L}(\mathbb{R})$ are in the class of Zames-Falb multipliers $\mathscr{M}$ if their impulse responses are of the form

$$
m(t)=h_{0} \delta(t)-\sum_{i=1}^{\infty} h_{i} \delta\left(t-d_{i}\right)-h(t),
$$

where $\delta$ is the Dirac delta function, $\sum_{i=1}^{\infty} h_{i}<\infty, h \in \mathscr{L}_{1}$, $d_{i} \in \mathbb{R}, \forall i \in \mathbb{N}^{+}$and the following conditions hold:

1) $\|h\|_{1}+\sum_{i=1}^{\infty}\left|h_{i}\right|<h_{0}$,

2) $h(t) \geq 0 \forall t \in \mathbb{R}^{+}$and $h_{i} \geq 0, \forall i \in \mathbb{N}^{+}$.
Theorem 1 (Zames-Falb theorem [8], [9]): Consider the feedback system in Fig. 1 with $G_{n} \in \mathbf{R H}_{\infty}$ and $\phi \in S[0, k]$. Suppose there exists a multiplier $M \in \mathscr{M}$ satisfying

$$
\Re\{M(j \omega)(1+k G(j \omega))\} \geq \varepsilon \quad \forall \omega \in \mathbb{R}^{+},
$$

where $\varepsilon>0$. Then the feedback interconnection is $\mathscr{L}_{2}$-stable. Condition in (1) is equivalent to

$$
\angle M(j \omega)+\angle(1+k G(j \omega)) \in(-90,90) \quad \forall \omega \in \mathbb{R}^{+},
$$

where the phase is given in degrees.

The stability theorem is also valid for time delayed plants. Several searches for Zames-Falb multipliers have been proposed in [10]-[14]. In this paper, because the plant has a delay, it is natural to use the class of irrational Zames-Falb multipliers discussed in [10]. We will show that either a onedelay multiplier, i.e.

$$
M_{d}(j \omega)=1-\lambda e^{-j d \omega},
$$

where $0<\lambda<1, d \in \mathbb{R}$; or a two-delay multiplier, i.e.

$$
\begin{aligned}
M_{2 d}(j \omega)=M_{d}(j \omega)+ & \mu M_{\hat{d}}(j \omega)= \\
& 1-\lambda_{1} e^{-j d \omega}+\mu\left(1-\lambda_{2} e^{-j \hat{d} \omega}\right),
\end{aligned}
$$

where $\mu>0$, is enough to show stability.

\section{Time delayed second order systems}

In this paper, we consider the case of a second order plant with no zero. Without loss of generality, we restrict our attention to the class $\mathscr{G}^{2}$ given by

$$
G_{ \pm, \xi, T_{d}}(s)= \pm \frac{e^{-s T_{d}}}{s^{2}+2 \xi s+1},
$$

where $T_{d} \geq 0, \xi>0$. As we will keep negative feedback in our analysis, we must consider both positive and negative cases.

In this paper, we consider the following:

Conjecture 2: Suppose $G \in \mathscr{G}^{2}$. Then either the off-axis circle criterion can be used to verify the Kalman conjecture, or there exists either a one-delay multiplier $M_{d} \in \mathscr{M}$ or a two-delay multiplier $M_{2 d} \in \mathscr{M}$ which satisfies

$$
\Re\left\{M_{d(\text { or } 2 d)}(j \omega)\left(1+k_{N} G(j \omega)\right)\right\} \geq 0 \quad \forall \omega \in \mathbb{R}^{+},
$$

where $k_{N}$ is the Nyquist gain of $G$.

Remark 1: The existence of a multiplier $M$ satisfying inequality (6) for $k_{N}$ implies that for any $0<k<k_{N}$ there is an $\varepsilon>0$ such that (1) is also satisfied with the same $M$; see Section 6.1 in [9] for further details. This in turn is sufficient to verify the Kalman conjecture for $G$.

\section{SYSTEMS WITH ZERO DAMPING RATIO}

In this section, we restrict our attention to the following two plants:

$$
G_{+, T_{d}}(s)=\frac{e^{-s T_{d}}}{s^{2}+2 \xi s+1}, \text { and } G_{-, T_{d}}(s)=\frac{-e^{-s T_{d}}}{s^{2}+2 \xi s+1},
$$

where $\xi \rightarrow 0^{+}$. This seems to be the most challenging case, as the phase of $1+k_{N} G$ has at least two changes of $180^{\circ}$. But in this case an analytic value of the Nyquist gain $k_{N}$ can be obtained. 


\section{A. Nyquist Gain}

It is easy to check the following via the Nyquist criterion:

a) For $G_{+, T_{d}}(s)$ : the analytic expression for the Nyquist value is given by

$$
k_{N}= \begin{cases}0 & \text { if } n-1 \leq T_{d} / \pi \leq n \\ 1-n^{2} \pi^{2} / T_{d}^{2} & \text { if } n \leq T_{d} / \pi \leq \sqrt{n^{2}+n+1 / 2} \\ (n+1)^{2} \pi^{2} / T_{d}^{2}-1 & \text { if } \sqrt{n^{2}+n+1 / 2} \leq T_{d} / \pi \leq n+1\end{cases}
$$

where $n \geq 1$ is an odd number.

b) For $G_{-, T_{d}}(s)$ : the analytic expression for the Nyquist value is given by

$$
k_{N}= \begin{cases}1 & \text { if } 0 \leq T_{d} / \pi \leq 1 / \sqrt{2}, \\ \pi^{2} / T_{d}^{2}-1 & 1 / \sqrt{2} \leq T_{d} / \pi \leq 1 \\ 0 & \text { if } n-1 \leq T_{d} / \pi \leq n \\ 1-n^{2} \pi^{2} / T_{d}^{2} & \text { if } n \leq T_{d} / \pi \leq \sqrt{n^{2}+n+1 / 2}, \\ (n+1)^{2} \pi^{2} / T_{d}^{2}-1 & \text { if } \sqrt{n^{2}+n+1 / 2} \leq T_{d} / \pi \leq n+1,\end{cases}
$$

where $n \geq 2$ is an even number.

\section{B. Slope of the Nyquist Plot}

The use of the phase at crossing frequencies requires an analysis of the slope of the tangent to the Nyquist plot at each frequency. Note that slope of the Nyquist plot of $1+k G$ for $k>0$ is the same as the slope of the Nyquist plot of $G$, so we will study the latter. For the sake of simplicity, let $x$ and $y$ denote the real and complex values of the Nyquist plot of $G_{ \pm}$, i.e.

$$
\begin{gathered}
x(\omega)=\mathfrak{R}\{G(j \omega)\}= \pm \cos \left(\omega T_{d}\right) /\left(1-\omega^{2}\right), \\
y(\omega)=\mathfrak{I}\{G(j \omega)\}=\mp \sin \left(\omega T_{d}\right) /\left(1-\omega^{2}\right) .
\end{gathered}
$$

Then the slope of the Nyquist plot is given by

$$
\begin{aligned}
\frac{d y}{d x}(\omega)=\frac{\frac{d y}{d \omega}(\omega)}{\frac{d x}{d \omega}(\omega)}= & \\
& \frac{\mp T_{d}\left(1-\omega^{2}\right) \cos \left(\omega T_{d}\right) \mp 2 \omega \sin \left(\omega T_{d}\right)}{\mp T_{d}\left(1-\omega^{2}\right) \sin \left(\omega T_{d}\right) \pm 2 \omega \cos \left(\omega T_{d}\right)}
\end{aligned}
$$

This simplifies at the crossing frequencies for which $\sin \left(\omega_{c} T_{d}\right)=0$, and we may say

$$
\frac{d y}{d x}\left(\omega_{c}\right)=-\frac{T_{d}\left(1-\omega_{c}^{2}\right)}{2 \omega_{c}} .
$$

Equation (9) also provides the slope of the asymptote of the Nyquist plot of $G$ at $\omega=1$, specifically

$$
\lim _{\omega \rightarrow 1} \frac{d y}{d x}(\omega)=-\tan \left(T_{d}\right) .
$$

C. Critical Frequencies $1+k_{N} G(j \omega)$

The development of a suitable multiplier for $\tilde{G}(j \omega)=$ $1+k_{N} G(j \omega)$ is based on the analysis of up to three critical frequencies. These critical frequencies are:

1) Nyquist Frequency $\omega_{N}$ : Let us define the Nyquist frequency, $\omega_{N}$, as the frequency at which the left-most crossing of negative real axis by the Nyquist plot of $G$ occurs, i.e. $G\left(j \omega_{N}\right)=-1 / k_{N}$. Then there is a change of $180^{\circ}$ degrees in the phase of $\tilde{G}$, which puts an equality constraint on the multiplier given by

$$
\angle M\left(j \omega_{N}\right)=-\left(\angle \tilde{G}\left(j \omega_{N}^{+}\right)+\angle \tilde{G}\left(j \omega_{N}^{-}\right)\right) / 2 .
$$

2) $\omega=1:$ As $\omega \rightarrow 1$, the gains of both $G(j \omega)$ and $\tilde{G}(j \omega)$ tend to infinity. In particular the Nyquist plot of $\tilde{G}(j \omega)$ has an asymptote at $\omega=1$. There is a $180^{\circ}$ change in the phase of $\tilde{G}(j \omega)$ which puts another equality constraint on the multiplier given by

$$
\angle M(j 1)=-\left(\angle \tilde{G}\left(j 1^{+}\right)+\angle \tilde{G}\left(j 1^{-}\right)\right) / 2 .
$$

3) 2nd Critical Crossing Frequency $\widehat{\omega}$ : If $\omega_{N}<1$, let $\widehat{\omega}$ be the lowest value of $\omega>1$ such that $\mathfrak{I}[G(j \omega)]=0$. Similarly if $\omega_{N}>1$ let $\widehat{\omega}$ be the highest value of $\omega<1$ such that $\mathfrak{I}[G(j \omega)]=0$. In general there is an inequality constraint on the phase of the multiplier at $\widehat{\omega}$ and the smaller the distance $\tilde{G}(\widehat{\omega})-\tilde{G}\left(\omega_{N}\right)$ the tighter the constraint. For some values of delay this distance is equal to zero, i.e. $\widehat{\omega}$ is a second Nyquist frequency; in such cases there is also an equality constraint on the phase of the multiplier at $\widehat{\omega}$ given by

$$
\angle M(j \widehat{\omega})=-\left(\angle \tilde{G}\left(j \widehat{\omega}^{+}\right)+\angle \tilde{G}\left(j \widehat{\omega}^{-}\right)\right) / 2 .
$$

This happens when the $n^{\text {th }}$ crossing at $\omega=\frac{n \pi}{T_{d}}<1$ and $(n+1)^{t h}$ crossing at $\omega=\frac{(n+1) \pi}{T_{d}}>1$ coincide. This occurs when the delay takes the value

$$
T_{d, n}^{*}=\pi \sqrt{n^{2}+n+1 / 2}, \text { for some } n .
$$

\section{Phase Properties of $\tilde{G}(j \omega)$}

Here we analyse the geometrical properties of $\tilde{G}(j \omega)$ and identify the values for the critical frequencies with different time delays. We focus on the interval $T_{d} \in(0,2 \pi]$ as this is enough to illustrate the behaviour of all the different phenomena that can occur. The interval is split into the following subintervals:

1) Subinterval 1: $0<T_{d} / \pi \leq 1 / 2$,

2) Subinterval 2: $1 / 2<T_{d} / \pi \leq \sqrt{1 / 2}$,

3) Subinterval 3: $1 / \sqrt{2}<T_{d} / \pi \leq 1$,

4) Subinterval 4: $1<T_{d} / \pi \leq \sqrt{5 / 2}$,

5) Subinterval 5: $\sqrt{5 / 2}<T_{d} / \pi \leq 2$.

As discussed in Section III-A, we are concerned with $G_{-, T_{d}}$ for subintervals 1,2 , and 3 and with $G_{+, T_{d}}$ for subintervals 4 and 5 .

Subinterval 1: On this subinterval the off-axis circle criterion can be used to verify the Kalman conjecture, since the Nyquist plot of $\tilde{G}(j \omega)$ with $\omega>0$ lies to the right of a line through the origin with slope $-\tan \left(T_{d}\right)$.

Specifically, when $T_{d} \in(0, \pi / \sqrt{2}], \omega_{N}=0, k_{N}=1$ and the phase of $\tilde{G}\left(j 0^{+}\right)$is $90^{\circ}$. As $\omega \rightarrow 1$ the Nyquist plot of $\tilde{G}(j \omega)$ approaches an asymptote with slope $-\tan \left(T_{d}\right)$ that crosses the real axis at $1-\frac{T_{d}}{2 \sin T_{d}}$. Since $\xi>0$ (we are considering the case $\xi \rightarrow 0^{+}$) the Nyquist plot sweeps clockwise as the frequency changes from $1^{-}$to $1^{+}$. The frequency of the second crossing is $\widehat{\omega}=\frac{\pi}{T_{d}}>1$, and the second crossing of the negative real axis is at $\tilde{G}(j \hat{\omega})=1-\frac{T_{d}^{2}}{\pi^{2}-T_{d}^{2}}>1-\frac{T_{d}}{2 \sin T_{d}}$.

Since the slope of the line in the off-axis circle criterion is negative, it follows that there exists an appropriate RC multiplier [15]. The RC multipliers can be phase-substituted by anticausal Zames-Falb multipliers [17]. 
When $T_{d}=\pi / 2$, the Nyquist plot of $\tilde{G}(j \omega)$ has a vertical asymptote at $x=1-\pi / 4$ and its real part is always positive. Hence the circle criterion is sufficient for absolute stability.

In summary, when $T_{d} \in(0, \pi / 2]$, the Kalman conjecture is verified.

Subinterval 2: When $\pi / 2<T_{d} \leq \pi / \sqrt{2}$ then as before $\omega_{N}=0$ and $k_{N}=1$. The formulae for $\widehat{\omega}, \tilde{G}(j \widehat{\omega})$ and the asymptote are also the same as for Subinterval 1. However the slope of the asymptote is positive so the maximum phase difference in the Nyquist plot of $\tilde{G}(j \omega)$ is greater than $180^{\circ}$. Hence, the off-axis circle criterion is not suitable, and it is necessary to construct a multiplier.

The phase of the multiplier at $\omega=0$ and $\omega=1$ is given by (12) and (13), respectively, i.e.

$$
\angle M(j 0)=0 ; \quad \angle M(j 1)=-90\left(1-2 \frac{T_{d}}{\pi}\right) .
$$

For low values of $T_{d}$ a one-delay parametrisation of the multiplier is sufficient. We remark that since $\angle \tilde{G}\left(j 0^{+}\right)=90^{\circ}$, so $\angle M\left(j 0^{+}\right)$should be negative, which indicates a negative value for $d$ in (3), i.e. an anticausal multiplier as with Subinterval 1 .

As $T_{d}$ increases so the value of $\tilde{G}(j \widehat{\omega})$ approaches $\tilde{G}\left(j \omega_{N}\right)=0$; the values coincide when $T_{d}=\pi / \sqrt{2}$. With this delay value the phase of the multiplier is fixed at $\widehat{\omega}$ by (14), i.e.

$$
\angle M(j \widehat{\omega})=90-\arctan \frac{\pi^{2}-T_{d}^{2}}{2 \pi} \text { with } T_{d}=\frac{\pi}{\sqrt{2}},
$$

where we have used (10) at $\omega T_{d}=\pi$. For larger values of delay $T_{d}$ it is useful to enrich the choice of multiplier by including two delays as in (4).

A summary of the conditions in this subinterval is given in Table I.

\begin{tabular}{|c|c|c|}
\hline Critical Frequency & Crossing & Phase of $M(j \omega)$ (degrees) \\
\hline$\omega_{N}=0$ & -1 & 0 \\
\hline 1 & N/A & $90\left(2 \frac{I_{d}}{\pi}-1\right)$ \\
\hline$\widehat{\omega}=\pi / T_{d}>1$ & $\frac{-T_{d}^{2}}{\pi^{2}-T d^{2}}$ & $\begin{array}{c}90-\arctan \frac{\pi^{2}-T_{d}^{2}}{2 \pi} \\
\text { (as } T_{d} \rightarrow \sqrt{1 / 2 \pi} \pi^{-}\end{array}$ \\
\hline
\end{tabular}

TABLE I: Subinterval 2 summary

Subinterval 3: In this case $\omega_{N}=\frac{\pi}{T_{d}}>1, k_{N}=\frac{\pi^{2}}{T_{d}^{2}}-1$, and $\widehat{\omega}=0$. For $T_{d} \rightarrow \sqrt{1 / 2} \pi^{+}$, the proximity between both crossing requires the flexibility provided by (4). As $\Re\left\{\tilde{G}\left(j 0^{+}\right)\right\}$ is a small positive number, so the initial condition of the multiplier, i.e. $d<0$, is still needed.

The conditions on the multiplier can be developed as previously, and are summarised in Table II. In this case there is no longer a requirement from the phase change of $\tilde{G}(j 0)$ that $\angle M(j 0)=0$. However this is a natural condition for any Zames-Falb multiplier.

As $T_{d} \rightarrow \pi, k_{N} \rightarrow 0$ and the search becomes simpler but the value of $\lambda$ in (3) approaches 1 . In the case $T_{d}=\pi, k_{N}=0$ so there is no available multiplier.

\begin{tabular}{|c|c|c|}
\hline Critical Frequency & Crossing & Phase of $M(j \omega)$ (degrees) \\
\hline$\omega_{N}=\pi / T_{d}>1$ & $\frac{-T_{d}^{2}}{\pi^{2}-T d^{2}}$ & $90-\arctan \frac{\pi^{2}-T_{d}^{2}}{2 \pi}$ \\
\hline 1 & N/A & $90\left(2 \frac{T_{d}}{\pi}-1\right)$ \\
\hline$\widehat{\omega}=0$ & -1 & 0 \\
\hline
\end{tabular}

TABLE II: Subinterval 3 summary

\begin{tabular}{|c|c|c|}
\hline Critical Frequency & Crossing & Phase of $M(j \omega)$ (degrees) \\
\hline$\omega_{N}=\pi / T_{d}<1$ & $\frac{-T_{d}^{2}}{T d^{2}-\pi^{2}}$ & $90-\arctan \frac{\pi^{2}-T_{d}^{2}}{2 \pi}$ \\
\hline 1 & N/A & $90\left(2 \frac{T_{d}}{\pi}-3\right)$ \\
\hline$\widehat{\omega}=2 \pi / T d>1$ & $\frac{-T_{d}^{2}}{4 \pi^{2}-T d^{2}}$ & $\begin{array}{c}90-\arctan \frac{4 \pi^{2}-T_{d}^{2}}{4 \pi} \\
\left(\operatorname{as~} T_{d} \rightarrow \sqrt{5 / 2} \pi^{-}\right)\end{array}$ \\
\hline
\end{tabular}

TABLE III: Subinterval 4 summary

Subinterval 4: When $\pi<T_{d} \leq \sqrt{5 / 2} \pi$, the summary of critical frequencies is provided by Table III

Similar to Subinterval 2, the two crossings are far apart from each other at the initial values of the interval, the multiplier should satisfy the phase requirements at $\omega_{N}$ and 1 , and multiple solutions of the multiplier in (3) can be obtained with $d \in \mathbb{R}$.

Similarly, when $T_{d} \rightarrow \sqrt{5 / 2} \pi^{+}$, especially at $T_{d}=$ $\sqrt{5 / 2} \pi, \mathfrak{R}\left\{G_{+, T_{d}}(j \widehat{\omega})\right\}=\Re\left\{G_{+, T_{d}}\left(j \omega_{N}\right)\right\}$, so both $\omega_{N}$ and $\widehat{\omega}$ are Nyquist frequencies. As a result, the phase of the multiplier is severely restricted at three frequencies and a two-delay multiplier in the form of (4) is needed.

Subinterval 5: This case is very similar to Subinterval 4, but the role of the critical frequencies changes a shown in Table IV.

\begin{tabular}{|c|c|c|}
\hline Critical Frequency & Crossing & Phase of $M(j \omega)$ (degrees) \\
\hline$\omega_{N}=2 \pi / T d>1$ & $\frac{-T_{d}^{2}}{4 \pi^{2}-T d^{2}}$ & $90-\arctan \frac{4 \pi^{2}-T_{d}^{2}}{4 \pi}$ \\
\hline 1 & N/A & $90\left(2 \frac{T_{d}}{\pi}-3\right)$ \\
\hline$\widehat{\omega}=\pi / T d>1$ & $\frac{-T_{d}^{2}}{T d^{2}-\pi^{2}}$ & $\begin{array}{c}90-\arctan \frac{\pi^{2}-T_{d}^{2}}{2 \pi} \\
\left(\text { as } T_{d} \rightarrow \sqrt{5 / 2} \pi^{+}\right)\end{array}$ \\
\hline
\end{tabular}

TABLE IV: Subinterval 5 summary

As previously discussed, the search requires the two delay multiplier given by (4) when $T_{d}$ is slightly larger than $\sqrt{5 / 2} \pi$, as the 3 constraints are significant. When $T_{d}$ approaches $2 \pi$, the problem become easier and the multiplier parametrised by (3) is enough to show stability.

When $T_{d}=2 \pi, k_{N}=0$; hence no multiplier is required.

\section{E. Selected Multipliers}

The analysis carried out in the previous section provides the essential properties so multipliers for different time delays can be found. We show several examples covering different scenarios. Firstly, we focus our attention in the interval $[0,2 \pi]$. Secondly, we show the existence of the multiplier for critical cases with higher time-delay.

1) $T_{d} \in[0,2 \pi]$ : Some feasible multipliers for plants with $T_{d} \in[0,2 \pi]$ are listed in Table V. For each case we are able to find a multiplier and hence verify the Kalman conjecture. We avoid the interval $[0, \pi / 2]$ as the off-axis circle criterion is sufficient on this subinterval. 


\begin{tabular}{|c|c|c|c|}
\hline Ex. & $T_{d}$ & $k_{N}$ & Suitable Multipliers \\
\hline 1 & 2.00 & 1 & $M_{d}=1-0.5448 e^{4.4000 s}$ \\
\hline 2 & $\sqrt{0.5} \pi$ & 1 & $\begin{array}{c}M_{2 d}=1-0.7886 e^{-31.6411 s} \\
+8\left(1-0.9988 e^{4.4438 s}\right)\end{array}$ \\
\hline 4 & 2.50 & 0.5791 & $M_{d}=1-0.9996 e^{5.0006 s}$ \\
\hline 6 & 3.13 & 0.0074 & $M_{d}=1-0.99999905 e^{6.2608 s}$ \\
\hline 7 & 3.15 & 0.0053 & $M_{d 1}=1-0.9999647 e^{-6.2751 s}$ \\
\hline 9 & $1.5 \pi$ & 0.5556 & $\begin{array}{c}M_{d 1}=1-0.8326 e^{-6.2832 s} \\
M_{d 2}=1-0.8330 e^{3.1416 s}\end{array}$ \\
\hline 11 & $\sqrt{2.5} \pi$ & 0.6000 & $\begin{array}{c}M_{2 d}=1-0.9280 e^{-6.2878 s} \\
+0.02181\left(1-0.9365 e^{23.5192 s}\right.\end{array}$ \\
\hline 12 & 4.97 & 0.5983 & $\begin{array}{c}M_{d 1}=1-0.9412 e^{-6.2997 s} \\
M_{d 2}=1-0.9972 e^{9.9407 s}\end{array}$ \\
\hline 14 & 6.27 & 0.0042 & $\begin{array}{c}M_{d 1}=1-0.9999 e^{-6.2899 s} \\
M_{d 2}=1-0.9999 e^{6.2635 s}\end{array}$ \\
\hline
\end{tabular}

TABLE V: Feasible multipliers for different $T_{d} \in[0,2 \pi]$. Figures and code for all examples are available in our website: https://goo.gl/BLuaNK.

It is instructive to consider Examples 2 and 11 in detail. These correspond to the values of $T_{d}$ on the interval $(0,2 \pi]$ where $\tilde{G}\left(j \omega_{N}\right)=\tilde{G}(j \widehat{\omega})$.

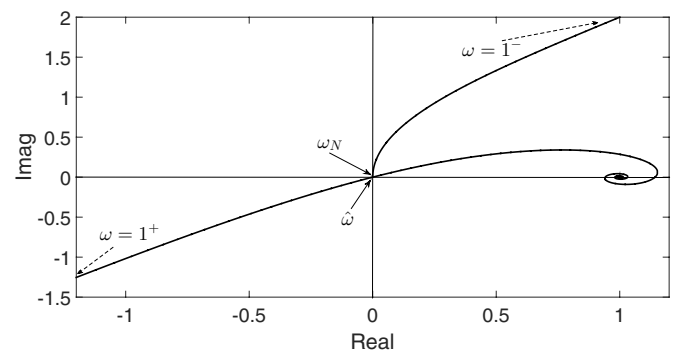

Fig. 2: Nyquist plot of $\tilde{G}(j \omega)$ of of Ex.2

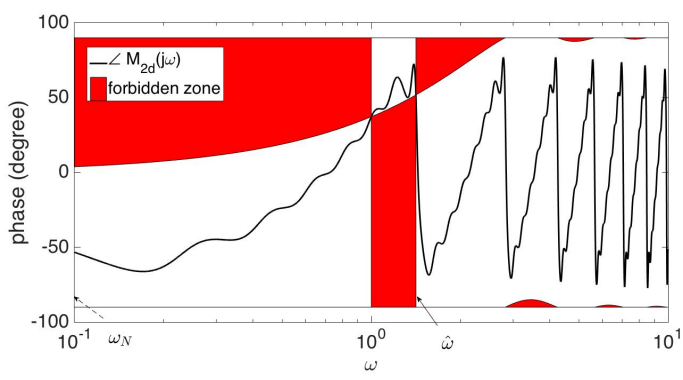

Fig. 3: $\angle M_{2 d}(j \omega)$ with forbidden zone of Ex.2

Fig 2 shows the Nyquist plot of $\tilde{G}(j \omega)$ for Example 2 $\left(T_{d}=\sqrt{1 / 2} \pi\right)$. This is the limiting case between Subintervals 2 and 3. Fig 3 shows the phase requirements for the multiplier, where the red regions are the forbidden zones. In particular there is an equality constraint on the multiplier at $\omega=1$ and $\omega=\sqrt{2}$ (there is a further equality constraint at $\omega=0$, not shown). Similar diagrams appear in [18]-[21] albeit in [19]-[21] with ratio between imaginary and real parts rather than phase shown on the vertical axis. In [21] such diagrams are named "Lipatov plots" after [19], [20].

Example $11\left(T_{d}=\sqrt{5 / 2} \pi\right)$ is the limiting case between Subinterval 4 and 5 where the multiplier should satisfy the

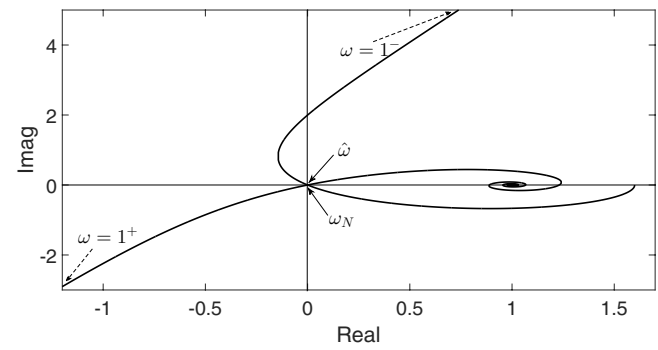

Fig. 4: Nyquist plot of $\tilde{G}(j \omega)$ of Ex.11

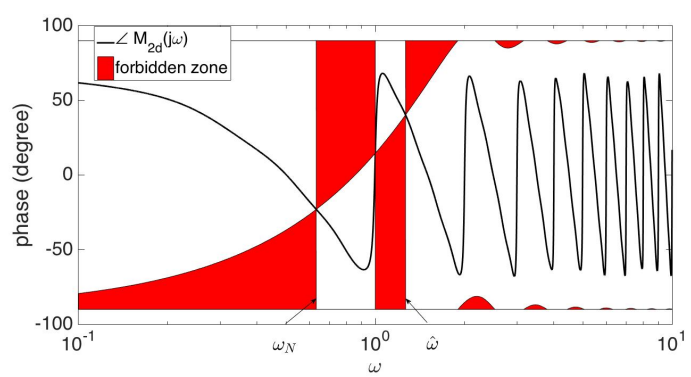

Fig. 5: $\angle M_{2 d}(j \omega)$ with forbidden zone of Ex.11

phase requirement at three critical frequencies (see Figs 4 and 5). Similar to the discussion above, the phase of the multiplier passes through each intersection of the forbidden zone at each frequency, so the phase requirements in (12) (13) (14) hold, and the multiplier is valid.

2) Longer time delays: For $T_{d}>2 \pi$ we limit our analysis the most challenging cases, where the two crossing at frequencies $\omega_{N}$ and $\widehat{\omega}$ overlap at the same point. The results are listed in Table VI.

\begin{tabular}{|c|c|c|c|}
\hline$n$ & $T_{d, n}^{*}$ & $k_{N}$ & Suitable Multipliers \\
\hline 1 & 4.9673 & 0.6000 & $\begin{array}{c}M_{2 d}=1-0.9280 e^{-6.2878 s} \\
+0.02181\left(1-0.9365 e^{23.5192 s}\right)\end{array}$ \\
\hline 2 & 8.0095 & 0.3846 & $M_{d}=1-0.9990 e^{16.0187 s}$ \\
\hline 3 & 11.1072 & 0.2800 & $\begin{array}{c}M_{2 d}=1-0.7434 e^{-12.5210 s} \\
+12.8760\left(1-0.7434 e^{9.6934 s}\right)\end{array}$ \\
\hline 15 & 48.7200 & 0.0644 & $\begin{array}{c}M_{2 d}=1-0.6581 e^{-50.2446 s} \\
+4.3971\left(1-0.6581 e^{47.1955 s}\right)\end{array}$ \\
\hline 16 & 51.8601 & 0.0606 & $\begin{array}{c}M_{2 d}=1-0.6183 e^{-50.2369 s} \\
+3.1257\left(1-0.6183 e^{53.4832 s}\right)\end{array}$ \\
\hline 55 & 174.3655 & 0.0180 & $\left.\begin{array}{c}M_{2 d}=1-0.6424 e^{-175.9225 s} \\
+3.8448\left(1-0.6424 e^{172.8085 s}\right.\end{array}\right)$ \\
\hline 56 & 177.5069 & 0.0177 & $\begin{array}{c}M_{2 d}=1-0.6311 e^{-175.9219 s} \\
+3.4913\left(1-0.63114 e^{179.0920 s}\right)\end{array}$ \\
\hline 155 & 488.5202 & 0.0064 & $\left.\begin{array}{c}M_{2 d}=1-0.6387 e^{-490.0860 s} \\
+3.7235\left(1-0.6387 e^{486.9544 s}\right.\end{array}\right)$ \\
\hline 156 & 491.6618 & 0.0064 & $\begin{array}{c}M_{2 d}=1-0.6346 e^{-490.0859 s} \\
+3.5954\left(1-0.6346 e^{493.2376 s}\right)\end{array}$ \\
\hline
\end{tabular}

TABLE VI: Feasible multipliers for different $T_{d, n}^{*}$.

As demonstrated in the table, multipliers have been obtained, and the Kalman conjecture is true for these examples. In general as $T_{d, n}^{*}$ increases so $k_{N}$ decreases. However, the values of both $\lambda_{1}$ and $\lambda_{2}$ in (4) reduce and appear to converge to a limit around 0.63. Meanwhile, the values of both $d$ and $\hat{d}$ 
in (4) take values close to $T_{d, n}^{*}$ for relatively larger delays.

Once again, in all the cases we consider we are able to construct a suitable multiplier. Furthermore the search becomes easier as $T_{d}$ increases. In conclusion, the Kalman conjecture appears to be verified when $\xi \rightarrow 0^{+}$.

\section{Systems With Non-ZERo DAmping RAtios}

When the damping ratio is fixed at a value greater than 0 , the restriction on the phase of the multiplier at $\omega=1$ is less severe. In fact, as $\xi$ increases, the range of delays for which the off-axis circle criterion is suitable increases. We show in [7] that when $\xi>\frac{1}{\sqrt{2}}$ the off-axis circle criterion can be used to verify the Kalman conjecture for any delay.

However there may no longer be analytic expressions for $\omega_{N}, k_{N}, \widehat{\omega}, G(j \widehat{\omega}), T_{d, n}^{*}$ etc. Thus although matters may be simpler, we do not claim a formal proof of absolute stability for all cases.

Therefore, in this section, a few examples are provided with different $\xi<\frac{1}{\sqrt{2}}$ at time delays with two Nyquist frequencies (i.e. where $\tilde{G}(j \widehat{\omega})=\tilde{G}\left(j \omega_{N}\right)=0$ ). The results are listed in Table. VII.

\begin{tabular}{|c|c|c|c|}
\hline$\xi$ & $T_{d, 1}^{*}$ & $k_{N}$ & Feasible Multipliers \\
\hline 0.0001 & 4.9675 & 0.6001 & $\begin{array}{c}M_{2 d}=1-0.9271 e^{-6.2876 s} \\
+0.02245\left(1-0.9357 e^{23.5194 s}\right.\end{array}$ \\
\hline 0.001 & 4.9693 & 0.6009 & $M_{d}=1-0.9275 e^{-6.2877 s}$ \\
\hline 0.1 & 5.2140 & 0.6842 & $M_{d}=1-0.8782 e^{-6.5085 s}$ \\
\hline 0.4 & 6.9621 & 0.8993 & $M_{d}=1-0.6543 e^{-8.2613 s}$ \\
\hline 0.6 & 11.8303 & 0.9854 & $M_{d}=1-0.3757 e^{-13.1903 s}$ \\
\hline
\end{tabular}

TABLE VII: Feasible multipliers for different $T_{d, 1}^{*}$ with $\xi \neq 0$

As illustrated in the table, when $\xi$ is still close to zero, such as $\xi=0.0001$, the phase requirement of the multiplier at $\omega=1$ is not completely relaxed, so the multiplier structure as in last section is also needed. As $\xi$ increases, the condition is relaxed and, hence the structure of the multiplier can be simplified.

The results appear to confirm that the case $\xi \rightarrow 0^{+}$is the most challenging. As $\xi$ increases, $k_{N}$ also increases but the behaviour of the phase of $\tilde{G}(j \omega)$ allows simpler multipliers to be used.

\section{CONCLUSION}

The paper has analysed a wide range of second-order systems with time delay, and shown in each case that a Zames-Falb multiplier can be found at the Nyquist value. All the examples considered satisfy the Kalman conjecture.

The design of the multiplier is significantly different to the case without delay, where a Popov multiplier suffices [3]. Indeed for $0<T_{d}<\pi / \sqrt{2}$ the restriction on the multiplier at $\omega=0$ means a Popov multiplier cannot be used. We show that the complexity of the multiplier depends on the proximity between the two largest crossings of the Nyquist plot with the negative real axis. The lack of closed-form values for the Nyquist value impedes the development of a formal proof, but our numerical analysis seems to indicate that the structure of the multiplier is not limited by any phase limitation presented in [22].

It remains open whether there exists any system which satisfies the Kalman conjecture without delay but fails to satisfy the Kalman conjecture when delay is included. This remains the topic of further investigation.

\section{REFERENCES}

[1] R. E. Kalman, "Physical and mathematical mechanisms of instability in nonlinear automatic control systems," Transactions of ASME, vol. 79, pp. 553-566, 1957.

[2] A. I. Lurie and V. N. Postnikov, "On the stability theory of control systems," Russian Prikl. Matem. i Mekh., vol. 8, 1944.

[3] N. E. Barabanov, "On the Kalman problem," Sib. Math. J., vol. 29 , pp. 333-341, 1988.

[4] R. E. Fitts, "Two counterexamples to Aizerman's conjecture," IEEE Transactions on Automatic Control, vol. 11, pp. 553-556, 1966.

[5] G. A. Leonov, V. O. Bragin, and N. V. Kuznetsov, "Algorithm for constructing counterexamples to the Kalman problem," in Doklady Mathematics, 2010, vol. 82, pp. 540-542.

[6] V. Răsvan, "Delay independent and delay dependent Aizerman problem," in Unsolved Problems in Mathematical Systems and Control Theory. Princeton University Press, 2004, ch. 6.6, pp. 21-220.

[7] J. Zhang, H. Tugal, J. Carrasco, and W. P. Heath, "Absolute stability of systems with integrator and/or time delay via off-axis circle criterion," Control Systems Letters, vol. 2, no. 3, pp. 411 - 416, 2018.

[8] G. Zames and P. L. Falb, "Stability conditions for systems with monotone and slope-restricted nonlinearities," SIAM J. Control, vol. 6 , pp. 89-108, 1968.

[9] J. Carrasco, M. C. Turner, and W. P. Heath, "Zames-Falb multipliers for absolute stability: from O'Shea's contribution to convex searches,' European Journal of Control, vol. 28, pp. 1-19, 2016.

[10] M. Safonov and G. Wyetzner, "Computer-aided stability analysis renders Popov criterion obsolete," IEEE Transactions on Automatic Control, vol. 32, pp. 1128-1131, 1987.

[11] X. Chen and J. T. Wen, "Robustness analysis of LTI systems with structured incrementally sector bounded nonlinearities," in American Control Conference, Proceedings of the 1995, vol. 5, Jun 1995, pp. $3883-3887$ vol.5.

[12] X. Chen and J. Wen, "Robustness analysis for linear time-invariant systems with structured incrementally sector bounded feedback nonlinearities," Applied Mathematics and Computer Science, vol. 6, p. 623648, 1996.

[13] M. C. Turner, M. Kerr, and I. Postlethwaite, "On the existence of stable, causal multipliers for systems with slope-restricted nonlinearities,' IEEE Transactions on Automatic Control,, vol. 54, pp. 2697-2702, 2009.

[14] J. Carrasco, M. Maya-Gonzalez, A. Lanzon, and W. P. Heath, "LMI searches for anticausal and noncausal rational Zames-Falb multipliers," Systems \& Control Letters, vol. 70, pp. 17-22, 2014.

[15] Y.-S. Cho and K. S. Narendra, "An off-axis circle criterion for the stability of feedback systems with a monotonic nonlinearity," IEEE Transactions on Automatic Control, vol. 13, pp. 413-416, 1968.

[16] W. P. Heath and J. Carrasco, "Global asymptotic stability for a class of discrete-time systems," in European Control Conference, 2015.

[17] J. Carrasco, W. P. Heath, and A. Lanzon, "Equivalence between classes of multipliers for slope-restricted nonlinearities," Automatica, vol. 49, no. 6, pp. 1732 - 1740, 2013.

[18] M. I. Freedman, "Phase function norm estimates for stability of systems with monotone nonlinearities," SIAM J. Control, vol. 10, no. 1, pp. 99-111, 1972.

[19] A. V. Lipatov, "Stability of stationary system with one nonlinear unit. i. fundamental theorems," Automation and Remote Control, vol. 43 , pp. 737-746, 1982.

[20] — "Stability of stationary system with one nonlinear unit. ii. geometrical criterion," Automation and Remote Control, vol. 43, pp. 865-871, 1982.

[21] D. Altshuller, Frequency domain criteria for absolute stability. Springer, 2013.

[22] S. Wang, J. Carrasco, and W. P. Heath, "Phase limitations of Zames-Falb multipliers," IEEE Transactions on Automatic Control, vol. 63, no. 4, pp. 947 - 959, 2018. 